This item was submitted to Loughborough's Research Repository by the author.

Items in Figshare are protected by copyright, with all rights reserved, unless otherwise indicated.

\title{
Large eddy simulation of high reynolds number jets with microjet injection
}

PLEASE CITE THE PUBLISHED VERSION

PUBLISHER

Published by the American Institute of Aeronautics and Astronautics, Inc. @ the author(s)

VERSION

VoR (Version of Record)

LICENCE

CC BY-NC-ND 4.0

REPOSITORY RECORD

Rife, M.E., and Gary J. Page. 2012. "Large Eddy Simulation of High Reynolds Number Jets with Microjet Injection”. figshare. https://hdl.handle.net/2134/9545. 
This item was submitted to Loughborough's Institutional Repository (https://dspace.lboro.ac.uk/) by the author and is made available under the following Creative Commons Licence conditions.

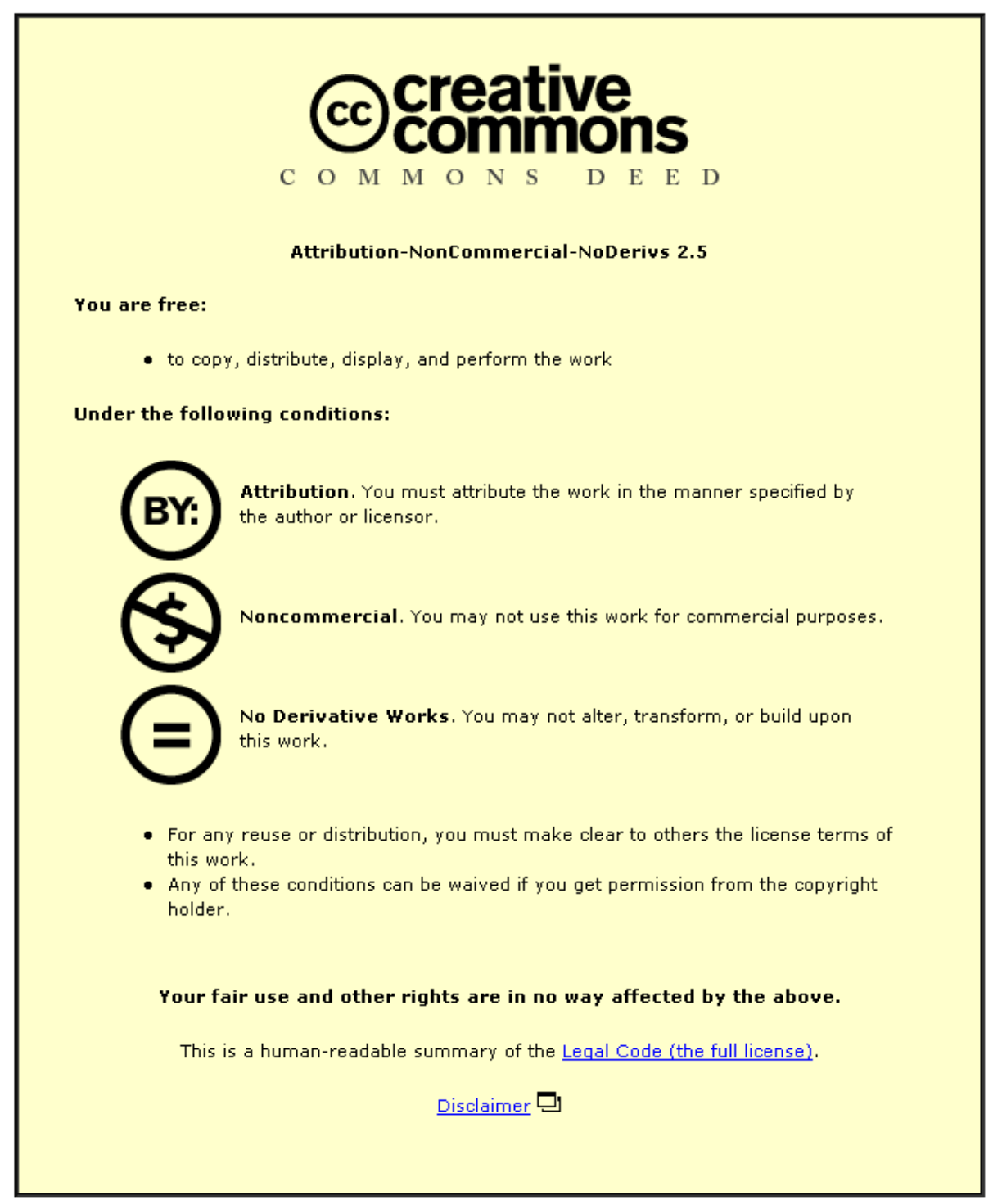

For the full text of this licence, please go to: http://creativecommons.org/licenses/by-nc-nd/2.5/ 


\title{
Large Eddy Simulation of High Reynolds Number Jets with Microjet Injection
}

\author{
M. E. Rife* and G. J. Page ${ }^{\dagger}$ \\ Department of Aeronautical \& Automotive Engineering, Loughborough University, Leicestershire, UK
}

\begin{abstract}
Large eddy simulations of two isothermal Mach 0.75 jets have been performed, one of a clean jet and one of the same jet fitted with eight equally spaced microjets. The microjets have a pressure ratio of 2.38, with a fully expanded Mach number of 1.19. The Reynolds number of the main jet in both simulations, based on the jet core velocity and diameter, is 1.3 million. The simulations were performed on a cylindrical, structured, multiblock mesh created for the clean round jet. The microjets are introduced as pressure inlet areas within the computational domain, so avoiding the complication of modelling the microjet feed pipes. Results of the clean jet simulation agree well with experimental data. The simulation shows the microjets penetrating into the jet core and disrupting the otherwise circular nature of the shear layer in the early flow development regions, though no change in mean flow variables is noticed by the end of the potential core. Two-point two-time correlation are performed on both cases and compared. The results show the microjets reduce the second and fourth order correlation amplitudes and turbulent lengthscales even at large axial locations downstream of the nozzle exit, where the effect of the microjets on the mean flow field is not present. This gives evidence as to how the microjets are able to reduce jet noise levels.
\end{abstract}

\section{Nomenclature}

$a_{o} \quad$ Speed of sound

$D_{j} \quad$ Jet Nozzle diameter

$D_{m j} \quad$ Microjet diameter

${ }^{k} L_{i j} \quad$ Eulerian integral lengthscale

LBM Lattice-Boltzmann Methodology

LES Large Eddy Simulation

$M \quad$ Mach number

$\dot{m} \quad$ Mass flow rate

MILES Monotonically Integrated Large Eddy Simulation

$p_{i j} \quad$ Compressive stress tensor

PIV Particle Image Velocimetry

$R_{i j} \quad$ Second order correlation function

$R_{i j k l} \quad$ Fourth order correlation function

$R e_{D_{j}} \quad$ Reynolds number based on jet diameter

$T_{i j} \quad$ Lighthill stress tensor

$u \quad$ Axial jet velocity

$U_{j} \quad$ Mean jet velocity

$\delta_{i j} \quad$ Kronecker delta

$\vec{\eta} \quad$ Cross correlation separation vector

$\rho \quad$ Density

$\tau \quad$ Cross correlation temporal separation

*Reasearch Student

${ }^{\dagger}$ Senior Lecturer, AIAA Member 


\section{Introduction}

Increasingly stricter international aircraft noise regulations and growing pressure from communities near airports are creating a requirement for quieter aircraft. With jet noise being a dominant contributing source of overall aircraft noise during take-off, a significant amount of work has focused on jet noise reduction. One method of noise reduction is the use of chevrons, or serrations, at the nozzle exit. These devices have been shown to reduce low frequency noise ${ }^{2-4}$ and are gradually being introduced to aircraft, such as the Boeing 787 Dreamliner. A similar method of noise reduction is the addition of multiple small jets ('microjets') near the nozzle exit and directed into the main jet flow. Theinteraction creates pairs of counter-rotating axial vortices in the high-speed side of the jet shear layer and a reduction of low frequency noise, with minimal high frequency lift. ${ }^{2,3,5}$ Microjets have an advantage over static devices such as chevrons in that they can be disabled when noise reduction is not necessary, such as cruise, thus eliminating fuel burn penalties during the majority of the mission.

Numerical simulations of microjets remain limited, with only a few published examples. ${ }^{1,8,11,14}$ Huet et $a l .{ }^{8}$ performed a study of continuous and pulsed microjets on hot and cold jets. The Reynolds numbers of the main jet in the isothermal and hot simulations were $1.0 \times 10^{6}$ and $3.2 \times 10^{5}$, respectively. The simulation technique used by Huet et al. ${ }^{8}$ was the Monotonically Integrated Large Eddy Simulation (MILES) approach, in which no subgrid model is used. In their simulations, they used 12 microjets, with the inflow boundary conditions for each microjet being applied to one hexahedral cell.

The acoustic results of Huet et al. overpredicted the baseline round nozzle noise by about $6 \mathrm{~dB}$. However, the noise reduction caused by continuous microjets was found to be about $1.5 \mathrm{~dB}$ for sideline noise, which agrees well with experimental results. ${ }^{8}$ It is possible that the discrepancy in the acoustic predictions is the result of the MILES approach and the noise reduction found in their simulations is the result of the microjets shifting part of the noise spectrum to higher frequencies. These higher frequencies are unable to be properly resolved by an excessively coarse grid coupled with the MILES scheme, thus causing the noise to be dissipated by the solver. Huet et al. acknowledge that the methodology does require refinement, but the results prove the feasibility of numerically simulating microjet nozzles for noise reduction and prediction, and further investigations will ensue.

Lew, Najafiyazdi, and Mongeau ${ }^{11}$ employed a Lattice-Boltzmann Methodology LES (LBM-LES), principally for the ease with which it can handle the complex geometries associated with microjet nozzles. The simulation was run at a Reynolds number of $1 \times 10^{5}$ with 18 microjets surrounding the nozzle. Grid refinement in the region of the microjets resulted in 20 cells across each microjet, significantly finer resolution than Huet et al. ${ }^{8}$ It is worth noting that as a result of using LBM-LES, the jet plume in their simulation has a distinct non-circular shape in both the clean nozzle and microjet simulations.

Acoustically, the results from Lew et al.'s simulations show good agreement with experimental results. The simulation overpredicts the clean nozzle noise by $1 \mathrm{~dB}$ for observer angles lower than $80^{\circ}$. However, it does accurately predict a reduction in noise of about $1.5 \mathrm{~dB}$, a similar result to that found in experiments of Alkislar ${ }^{2}$ and Castelain. ${ }^{5}$

Recently, a simulation including microjets was performed by Liu et al. ${ }^{14}$ They studied the effect of fluidic injection on imperfectly expanded supersonic jets. They simulate an underexpanded nozzle with 12 microjets.

The results of Liu et al. captured the longitudinal vortices caused by the penetrating flow from the microjets. When the mass flow ratio between the microjets and main jet was increased the size and strength of these vortices increased, as did the thickness of the shear layer. They found that microjets caused a reduction in shock-cell size and strength, with higher mass flow ratio causing greater reduction. Noise reductions were in agreement with experimental results, with near-field high frequency noise being increased. ${ }^{14}$

The present work is to perform numerical simulations of a high subsonic jet fitted with eight microjets for comparison to an equivalent clean jet. The microjet parameters are based on the experimental work of Alkislar et al. ${ }^{3}$ Investigation of the effect of microjets on the spatio-temporal correlation values are undertaken, in an effort to understand the means by which microjets reduce jet noise.

This paper will begin with a description of the computational method used in the simulations. Following this a description of the two-point, two-time correlations will be given. Mean flow results will be compared between experimental clean jet data and the clean and microjet simulations. Finally, spatio-temporal correlation data will be presented, with comparison to experimental data, where available. 


\section{Computational Methodology}

\section{A. Solver}

The computational solver is a finite volume pressure-based method on a multiblock structured grid. Whilst pressure-based solvers are typically used for low speed incompressible flows, the method has been extended to include compressibility using conservative variables and can efficiently compute high speed flows including shock waves. The spatial discretization is a high order upwind scheme (equivalent to QUICK) and for LES the limiter is disabled. A four-stage Runge-Kutta scheme is available for temporal discretization, but with the small time-steps used in LES it has been found that a single stage backward Euler method is more efficient and has no impact on accuracy. Parallelisation is achieved by mapping the grid blocks to individual processors and updating the interface ('halo') regions using message passing.

\section{B. Grid}

Simulations were performed on a cylindrical grid with $438 \times 102 \times 360$ (axial $\times$ radial $\times$ azimuthal) elements. This results in 15.1 million elements split into 11 blocks. The mesh extends $30 D_{j}$ downstream, $10 D_{j}$ in the radial direction, and $6 D_{j}$ upstream of the nozzle exit to include the nozzle geometry for increased accuracy of flow development. Figure 1 shows half of the cylindrical computational domain, clearly indicating the nodal bunching in the regions of the nozzle exit and shear layer. The grid geometry is based on the JEAN experimental nozzle and was originally used for clean jet simulations. A clean jet simulation has previously been run on this grid with excellent comparison to experimental results. ${ }^{9}$ The current calculations do include numerical boundary layer 'trips' within the nozzle prior to the contraction. The trips introduce weakly correlated turbulence near the wall which persists at a low level at the nozzle exit plane. Although this turbulence is weak, it causes a realistic generation of the initial shear layer and avoids the creation of unrealistic axisymmetric structures near the nozzle exit.

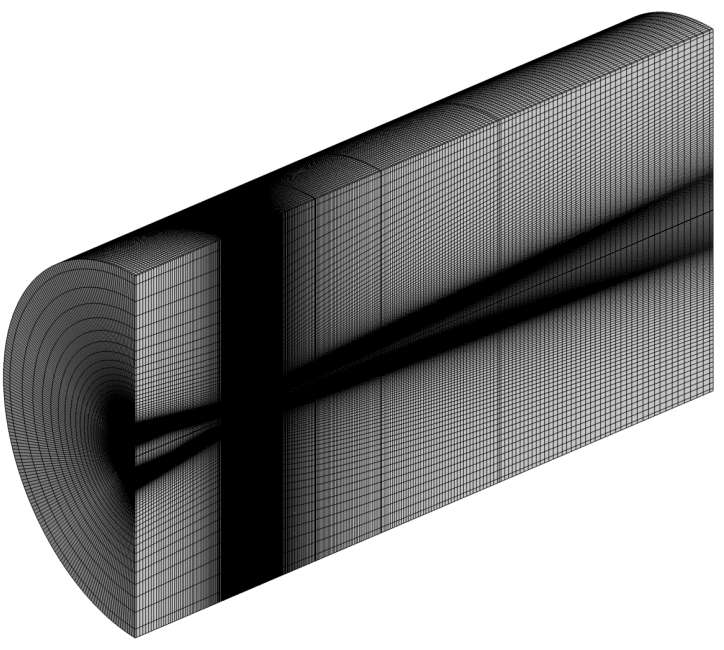

Figure 1. View of mesh used for simulations

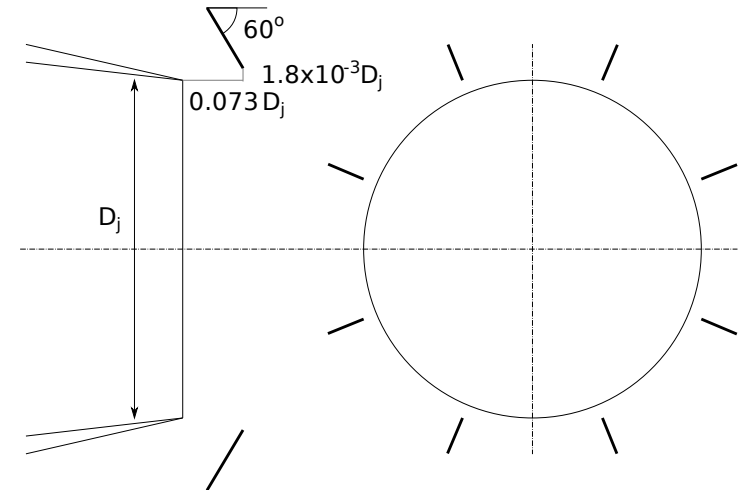

Figure 2. Schematic of microjet geometry

\section{Microjet Configuration}

Using the results of the clean jet simulation as a starting point, eight equally spaced microjets located just downstream of the nozzle exit were added to the simulation. The geometric layout of the microjets is shown in Figure 2. The microjets are equally spaced around the circumference of the jet nozzle and are located $0.073 D_{j}$ downstream from the exit plane and $1.8 \times 10^{-3} D_{j}$ radially outwards from the lip line. The thick black lines in Figure 2 serve only to indicate the location of the microjets. As a result of using an existing grid, the microjet resolution is restricted to $2 \times 2$ at their inlet, giving the microjets an equivalent diameter of $D_{m j}=0.021 D_{j}$. The mesh in the microjet region is shown in Figure 3, with the nozzle shown shaded grey. Iso-surfaces of Mach 0.35 serve to indicate the flow from the microjets whereas the mesh at a constant 
radial station is depicted (i.e. the mesh is not fixed to the iso-surface). The microjets were introduced to the simulation as pressure inlets located within the solution domain. By introducing the microjets in this way, the complex geometry associated with the microjet feed pipes around the nozzle is avoided. Furthermore, the inclusion of microjets in the simulation does not noticeably increase run time compared to the clean jet. The location of the microjets is such that the non-dimensional location is equivalent to Alkislar et al. ${ }^{3}$ The microjets are similarly inclined to the main jet axis by 60 degrees and they have a fully expanded Mach number of 1.19. The Reynolds number of the main jet and microjets are 1.3 million and 40,000 respectively (based upon jet velocity and diameter). The momentum flux ratio between the microjets and the main jet is 2.5 , and the mass flow ratio of each individual microjet to the main jet is $0.6 \times 10^{-3}$.

\section{Sampling}

In both cases (clean jet and microjet fitted), the time step for the simulation is approximately $5 \times 10^{-7} s$. Two simulations of each were run, the first consisting of 40,000 time steps (to be referred to as the short case), and the second having 80,000 time steps (referred to as the extended run case). At 80,000 time steps, the simulation time is equivalent to more than six flow through times. In all cases, mean and stress samples were taken at every tenth step and subsamples for correlation calculations were taken at 40 step intervals. For correlation calculations, 8 statistically independent slices at equivalent azimuthal locations were ensembled to aid in convergence. The result was 8,000 subsamples for the short case and 16,000 subsamples for the extended run case.

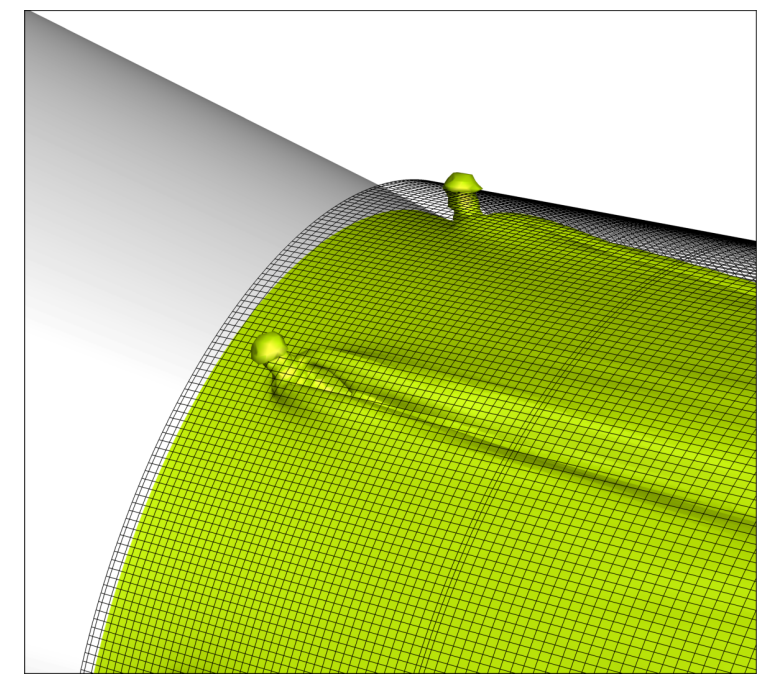

Figure 3. Detail of microjet injection. Isosurface of $M=0.35$.

\section{Correlations}

Through direct rearrangement of the Navier Stokes equations, Lighthill's acoustic analogy provides a means of treating a turbulent flow as a distribution of quadrupoles, the strength of which are determined as:

$$
T_{i j}=\rho v_{i} v_{j}+\left(p_{i j}-a_{o}^{2} \rho \delta_{i j}\right)
$$

where $\rho v_{i} v_{j}$ is the fluctuating Reynolds stress, $p_{i j}$ is the compressive stress tensor, and $a_{o}^{2}$ is the speed of sound squared. ${ }^{12,13}$ In an isothermal flow, such as those presently under investigation, only the first term on the right hand side becomes significant.

Karabasov et al. ${ }^{10}$ show the important relationship between the Lighthill stress tensor, $T_{i j}$, and twopoint, two-time correlations. An acoustic analogy based on information from these correlations has been shown to provide accurate results, when compared to experiments. Thus, a knowledge of how microjets affect the correlations may provide a means of modifying the jet noise model to take into account the microjets. The definitions of the two-point, two-time correlations used in this work have been put forth previously ${ }^{15,18}$ and are presented here for completeness. 
Let $\vec{x}$ indicate a reference point in space from which correlations will be measured with reference to at initial time, $t$. Then $\vec{\eta}$ represents a spatial separation vector and $\tau$ denotes temporal separation. For simplicity, A, B, and $\mathrm{C}$ represent specific coordinate pairs as follows:

$$
A=(\vec{x}, t), B=(\vec{x}+\vec{\eta}, t), C=(\vec{x}+\vec{\eta}, t+\tau)
$$

The the normalised second and fourth order correlations are given by:

$$
\begin{gathered}
R_{i j}(\vec{x}, \vec{\eta}, \tau)=\frac{\overline{\overline{u_{i}^{\prime}(A) u_{j}^{\prime}(C)}}}{\sqrt{\overline{\overline{u_{i}^{\prime}(A)^{2}}} \sqrt{\overline{u_{j}^{\prime}(B)^{2}}}}} \\
R_{i j k l}(\vec{x}, \vec{\eta}, \tau)=\frac{\overline{u_{i}^{\prime}(A) u_{j}^{\prime}(A) u_{k}^{\prime}(C) u_{l}^{\prime}(C)}-\left(\overline{\overline{u_{i}^{\prime}(A) u_{j}^{\prime}(A)}}\right)\left(\overline{\overline{u_{k}^{\prime}(B) u_{l}^{\prime}(B)}}\right)}{\left(\overline{u_{i}^{\prime}(A)^{4}}\right)^{\frac{1}{4}}\left(\overline{u_{j}^{\prime}(A)^{4}}\right)^{\frac{1}{4}}\left(\overline{u_{k}^{\prime}(B)^{4}}\right)^{\frac{1}{4}}\left(\overline{u_{l}^{\prime}(B)^{4}}\right)^{\frac{1}{4}}-\sqrt{\overline{u_{i}^{\prime}(A)^{2}}} \sqrt{\overline{u_{j}^{\prime}(A)^{2}}} \sqrt{\overline{u_{k}^{\prime}(B)^{2}}} \sqrt{\overline{\overline{u_{l}^{\prime}(B)^{2}}}}}
\end{gathered}
$$

It is possible to extract the Eulerian integral lengthscales from the second order correlations as below:

$$
{ }^{k} L_{i j}(\vec{x})=\int_{0}^{\infty} R_{i j}\left(\vec{x}, \eta_{k}, 0\right) d \eta_{k}
$$

where $k$ indicates the component of the direction vector along which the integration is calculated. For a limited field of view, such as in the present simulations, the integration is carried out to the first zero crossing of the correlation. This provides accurate results providing that the point is located within a sufficiently large domain to ensure capture of the first zero crossing.

It has been noted by Karabasov et al. ${ }^{9}$ that of the 21 unique $4^{\text {th }}$ order correlations only six have significant amplitude to be necessary for inclusion in an acoustic source description, ${ }^{10}$ those correlation functions being $R_{1111}, R_{1112}, R_{1212}, R_{1313}, R_{2222}$, and $R_{3333}$.

The goal of the present investigation is to determine what change, if any, the inclusion of microjets induces on the correlation distribution and amplitude of the affected jet.

\section{Results}

\section{A. Overall Flow Description}

Figure 4 shows instantaneous iso-surfaces of Mach 0.4 for the clean and microjet configuration, coloured by axial velocity. The nozzle surface is semi-transparent so that the change in colour indicates the nozzle exit plane and the rapid development of turbulence is apparent. The microjet injection location is in this early development phase, but does not appear to cause any significant qualitative change in the turbulence development.

Figure 5 shows the normalised centreline velocity profiles of the JEAN experimental data, ${ }^{6}$ the clean jet and the microjet case simulations. It should be noted that in the microjet case there is a small acceleration of the core velocity near the nozzle exit that lasts for about 0.5 jet diameters. The cause of this will be discussed below. Otherwise, the figure shows a good agreement with the experimental data for both simulations, with no noticeable change in the potential core length in the microjet case. By the end of the potential core, the difference between the the clean and microjet cases is indiscernible.

Radial velocity profiles at $x=1.0 D_{j}, 2.5 D_{j}$ and $5.0 D_{j}$ are shown in Figures $6(\mathrm{a})$-(c). For the simulation results, the values calculated in these plots are the result of averaging eight equivalent positions from around the jet. This was done to aid in statistical convergence of the flow variables. The clean jet simulation closely approximates the experimental data at all axial locations, however the velocity decay with radial location is slower in the simulation at all stations. In between the microjets the radial profiles show a slightly wider shear layer than the baseline for the first two axial locations, yet this difference is negligible by $x=5.0 D_{j}$. At $x / D_{j}=1.0$ there is a clear knee near $y / D_{j}=0.4$ where the wake of the microjet causes a reduction in the mean axial velocity. The change in shape of the profile caused by the presence of the microjets is absent by $2.5 D_{j}$ downstream, but a small reduction in the width of the jet core is still visible. By the most downstream location the profiles all show a similar shape, with no significant difference between them. 


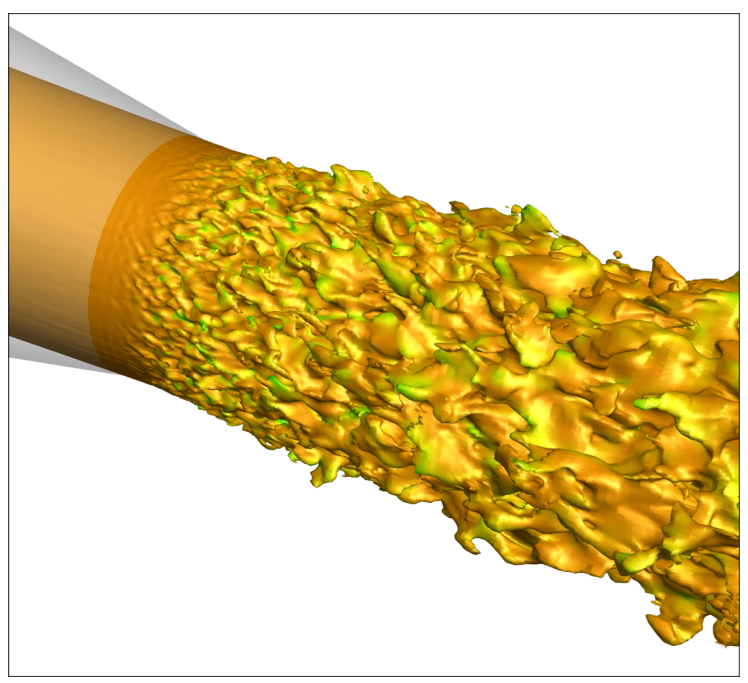

(a) Clean Jet

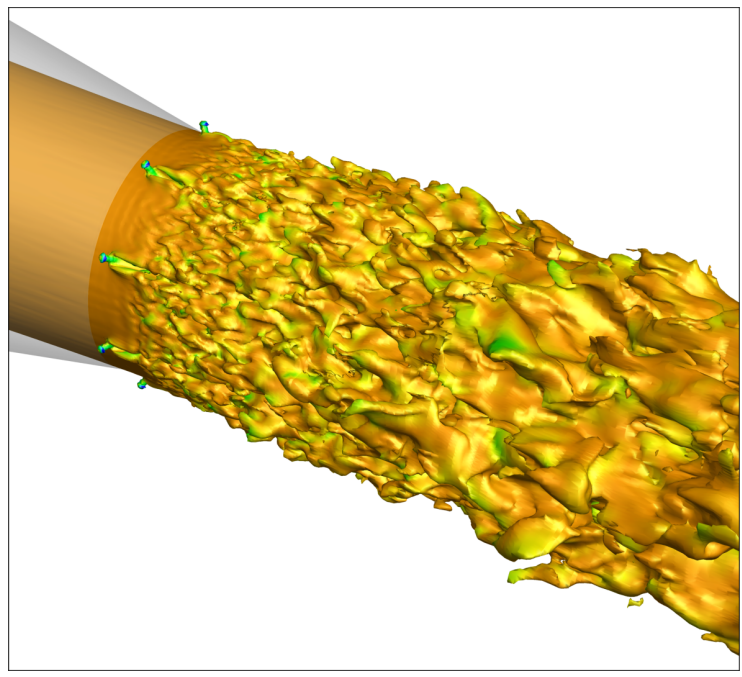

(b) Microjet

Figure 4. Instantaneous $M=0.4$ isosurface coloured by axial velocity

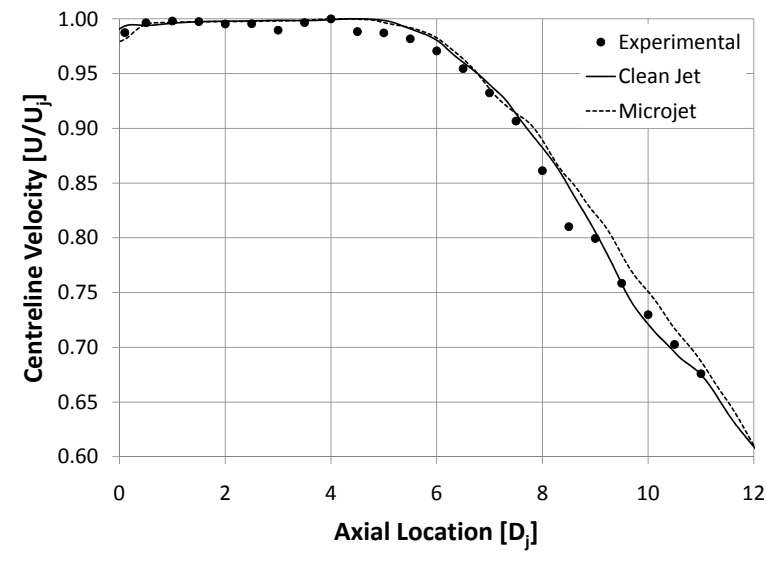

Figure 5. Normalised centreline velocities for experimental data and clean jet and microjet simulations

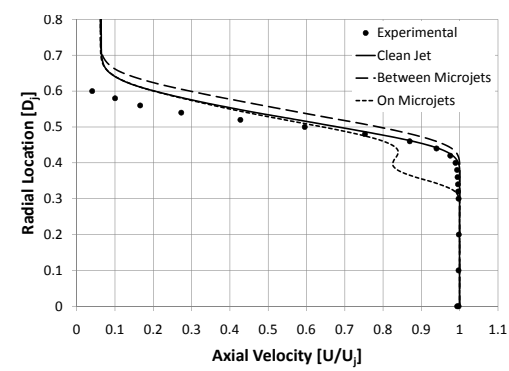

(a) Radial $x=1.0 D_{j}$

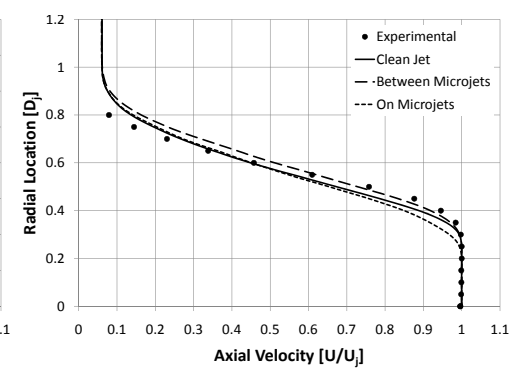

(b) Radial $x=2.5 D_{j}$

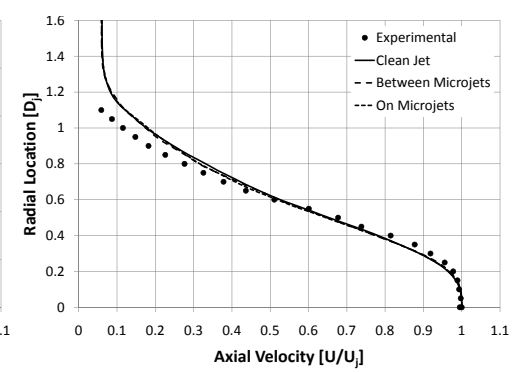

(c) Radial $x=5.0 D_{j}$

Figure 6. Velocity profiles at $x=1.0 D_{j}, 2.5 D_{j}$ and $5.0 D_{j}$ 


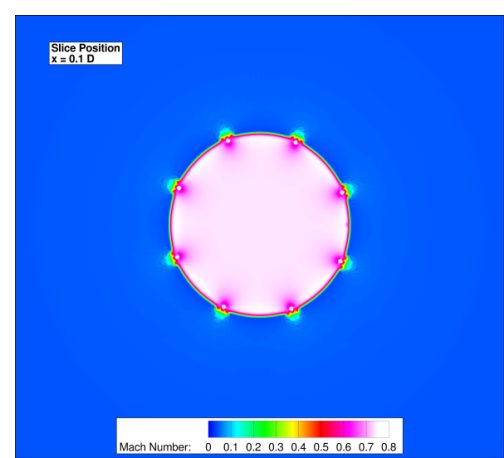

(a) Slice at $x=0.10 D_{j}$

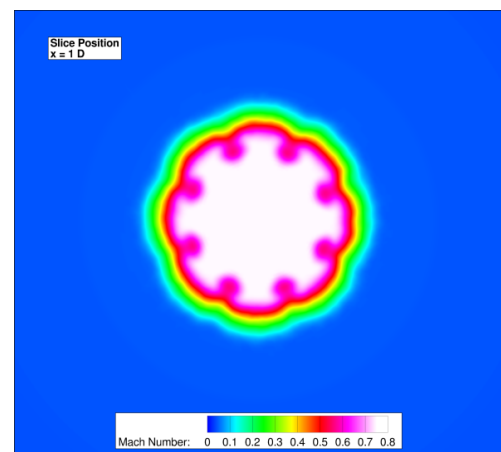

(d) Slice at $x=1.00 D_{j}$

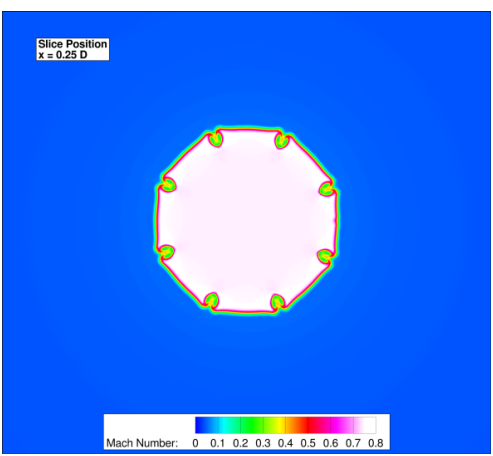

(b) Slice at $x=0.25 D_{j}$

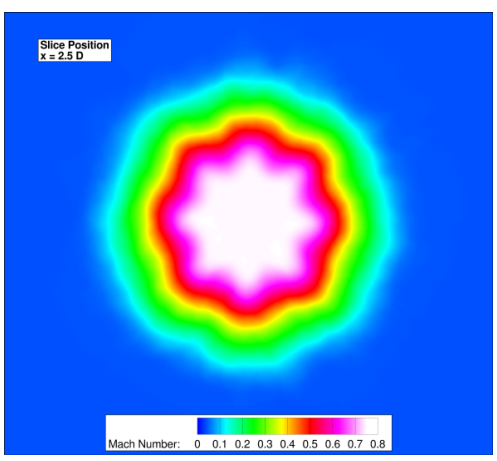

(e) Slice at $x=2.50 D_{j}$

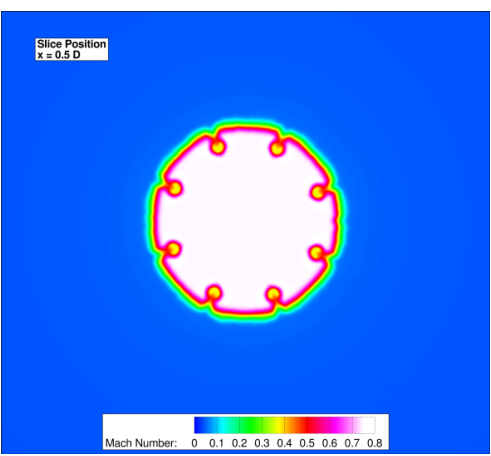

(c) Slice at $x=0.50 D_{j}$

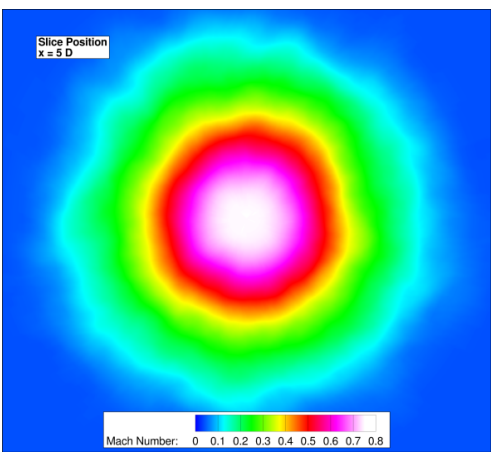

(f) Slice at $x=5.00 D_{j}$

Figure 7. Contours of mean Mach number for initial jet development.

Contours of mean Mach number are shown in shown in Figure 7 at four axial locations up to $5.0 D_{j}$ from the nozzle exit of the microjet case. It should be noted here that the data has not had any azimuthal averaging performed for the results shown in these figures and a small visualisation artifact of the block edge is seen at $0.5 D_{j}$ on the right side of the jet. Figure 7(a) shows the microjets first penetrating into the shear layer at $x=0.1 D_{j}$. By $0.25 D_{j}$ the microjets have pierced the shear layer each shedding a pair of counter-rotating vortices - the velocity field induced by the vortices has the curious property of distorting the circular jet shear layer into an octagonal shape composed of planar shear layers. In later plots, the microjet wakes grow and merge with the jet shear layer. As was noted from Figure 5 it can be seen from the shade of the jet core that acceleration occurs after microjet injection; this seems to be due to the microjets pinching the stream-tube, thus causing an inviscid acceleration. By $x / D_{j}=5.0$ the jet plume has returned to a circular shape. The effect of the microjets can not be seen in the velocity field of the jet at this late axial location.

Figure 8 shows the evolution of the turbulent kinetic energy normalised by $U_{j}^{2}$ behind the nozzle lip line. These results are an average of 8 equally separated lines, for all data. On the microjets, the turbulent kinetic energy demonstrates an unexpected behaviour within the first jet diameter downstream of the nozzle exit. The turbulent kinetic energy experiences a large peak around $x / D_{j}=0.25$, then drops to match the clean jet case just upstream of $0.5 D_{j}$, increasing at the same rate until $x=0.5 D_{j}$ at which point it plateaus for approximately half a diameter. By $x=1.0 D_{j}$ the turbulent kinetic energy in line with the microjets begins to decay at a similar rate to that of the clean case, consistently remaining at a lower level. Conversely, the turbulent kinetic energy behind a point between the microjets peaks at a larger value than the clean jet near $0.75 D_{j}$ downstream, but reaches a similar value as the clean case within a further jet diameter.

Axial slices colored by the turbulent kinetic energy for both the clean and microjet cases are displayed in Figure 9. Lines of axial velocity are provided for reference. A $x / D_{j}=0.25$ localised regions of high turbulent kinetic energy are seen either side of where the microjets inject into the shear layer. Between the microjets there is no visible effect on the kinetic energy. At $x / D_{j}=0.5$ these regions have grown in strength and size to include the region in line with the microjets. By one diameter downstream of the exit the the regions of high kinetic energy have dissipated. Still present at this point, however, are the plumes of low velocity and 


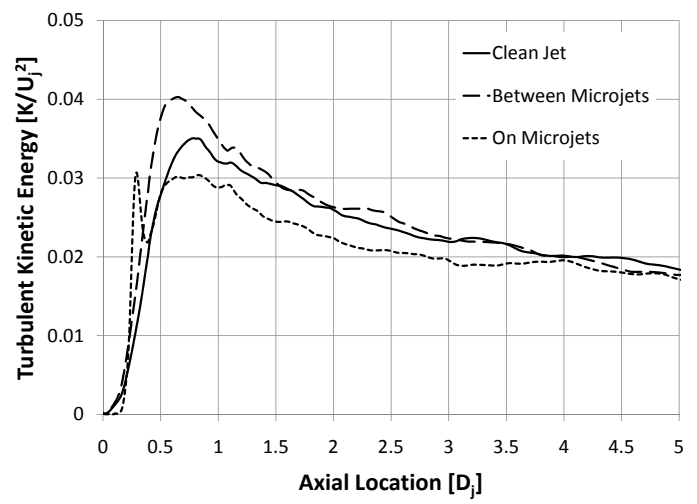

Figure 8. Normalised lipline turbulent kinetic energy values for clean jet and microjet simulations

increased turbulent kinetic energy in the wake of the microjets on the high speed side of the shear layer.

Figure 10 shows radial profiles of turbulent kinetic energy for the clean and microjet cases at $x / D_{j}=1.0$, 2.5 and 5.0. At one jet diameter downstream from the nozzle exit the effect of the microjets is still quite pronounced, increasing turbulence nearer the jet centreline. Figure 10 (a) indicates that the presence of the microjets also shifts the shear layer radially outward between the microjets, compared to the clean case. This effect remains present at $x=2.5 D_{j}$ while the thickening of the shear layer behind the microjets becomes significantly less pronounced. By $5.0 D_{j}$ downstream the shape of the profiles is similar in all three cases. The presence of the microjets has reduced the turbulent kinetic energy both behind and between the microjets at all axial locations, an effect not clear from Figure 9.

From the above, it is clear that the presence of microjets around the jet have an influence on the jet shear layer. To begin to understand the effect that these microjets have on the jet noise one can look at the spatio-temporal correlations.

\section{B. Correlation Results}

The second and fourth order correlation distributions of Pokora et al. ${ }^{16}$ and the short and extended clean jet runs at $x / D_{j}=1.5$ are plotted against $\tau 0.6 U_{j} / D_{j}$ in Figures 11 (a) \& (b), where $0.6 U_{j}$ is set as the convection speed for all cases. The fixed convection velocity allows for differences between the cases to be easily identified. A similar process of slice averaging as described in Section II D was used by Pokora et $a l$., but 36 slices were used in their work, giving 36,000 samples for their correlations. The location of the peak values for $\eta=0.2 D_{j}$ and $\eta=0.4 D_{j}$ shows the same convection velocity between the three simulations. The similarity in shape of the curves in both Figures 11(a) and 12(a) indicates similar turbulent scales and behaviour in all cases. In Figure 11(a) both simulations have a slightly lower peak value at $\eta=0.2 D_{j}$ than that of Pokora et al. However, this is reduced by $\eta=0.4 D_{j}$. Figure 11(b) shows good agreement between the extended run and the work of Pokora et al. Looking at the short run in Figure 11(a) we see that the convergence is not as strong as that of the extended run, an expected result. Overall, the agreement between the extended run and the work of Pokora et al. is good for the second and fourth order correlations.

Figures $12(\mathrm{a}) \&(\mathrm{~b})$ show the correlation distributions at $x / D_{j}=4.0$. Again, good correspondence between the extended run and the results from Pokora et al. is seen. In the $4^{\text {th }}$ order auto-correlations (i.e. $\eta=0 D_{j}$ ) the extended run deviates from Pokora et al.'s results from around $\tau 0.6 U_{j} / D_{j}=0.5$. However, the extended run results do remain above zero for the majority of the period.

The relative amplitude of the second order correlations at $x / D_{j}=4.0$ is compared to the experimental results of Harper-Bourne, ${ }^{7}$ Pokora's water tunnel data ${ }^{17}$ and Pokora el al.'s simulation in Table 1. Note that Harper-Bourne's data was collected using a hot-wire anemometer on a Mach 0.18 jet at a Reynolds number of $2 \times 10^{5}$. Conversely, Pokora's experimental data was obtained from PIV of a incompressible water jet $(\mathrm{Mach}=0)$ with a Reynolds number of $4 \times 10^{4}$. Pokora's incompressible experimental work consistently shows lower correlation values than Harper-Bourne. A very strong agreement between the experimental results of Harper-Bourne and the extended run are seen for $R_{11}, R_{22}$, and $R_{33}$. Between the extended run simulation and the work of Pokora et al. an equally good agreement is seen in the other correlation functions. The simulations show a consistent over-prediction compared to Pokora's experimental work. The 


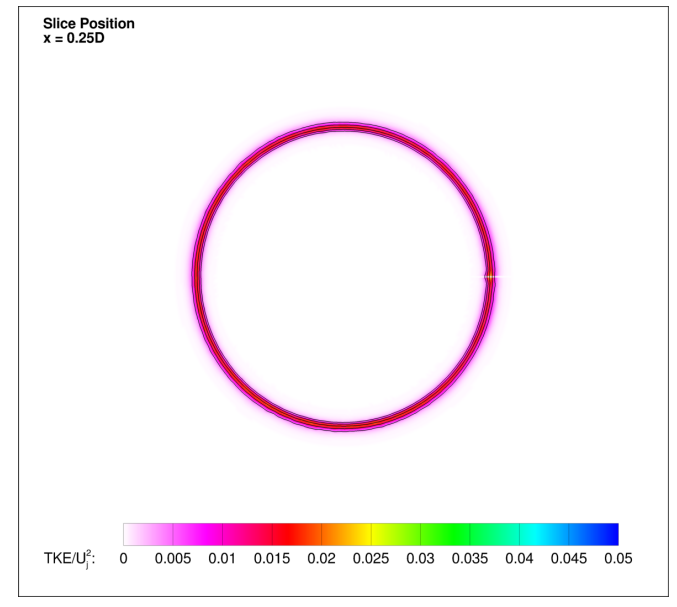

(a) Clean Jet $K / U_{j}^{2}$ at $x=0.25 D_{j}$

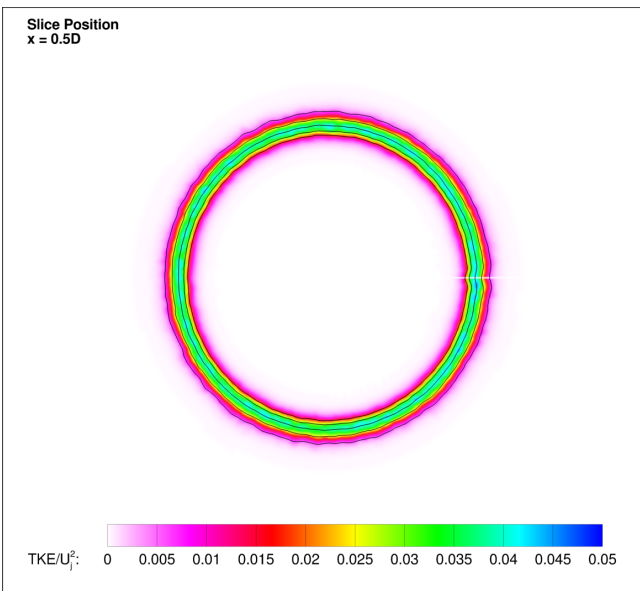

(c) Clean Jet $K / U_{j}^{2}$ at $x=0.5 D_{j}$

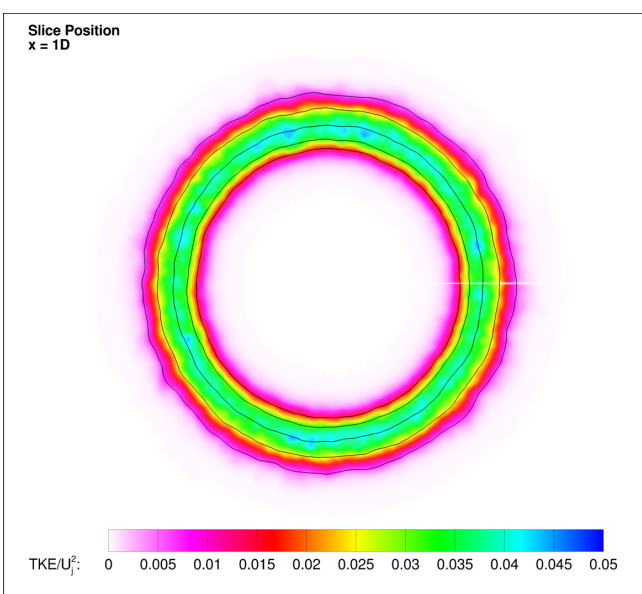

(e) Clean Jet $K / U_{j}^{2}$ at $x=1.0 D_{j}$

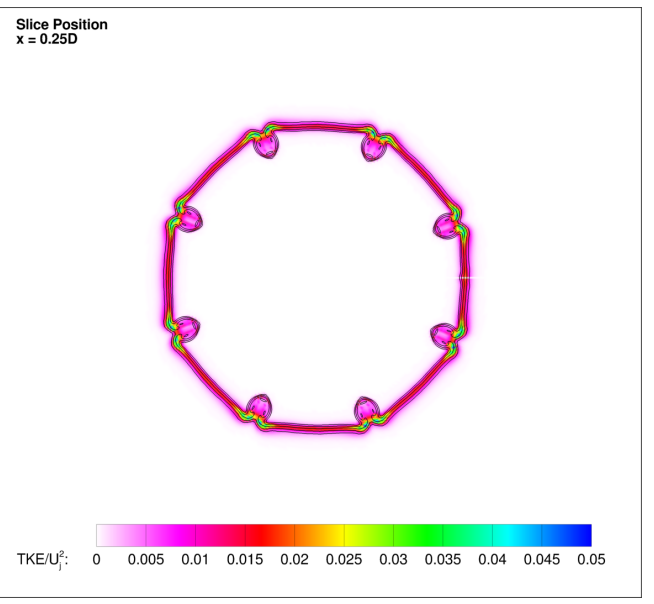

(b) Microjet case $K / U_{j}^{2}$ at $x=0.25 D_{j}$

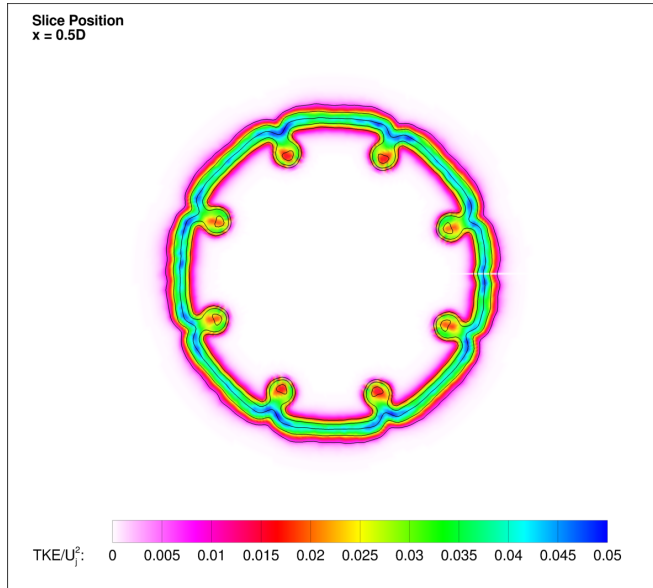

(d) Microjet Case $K / U_{j}^{2}$ at $x=0.5 D_{j}$

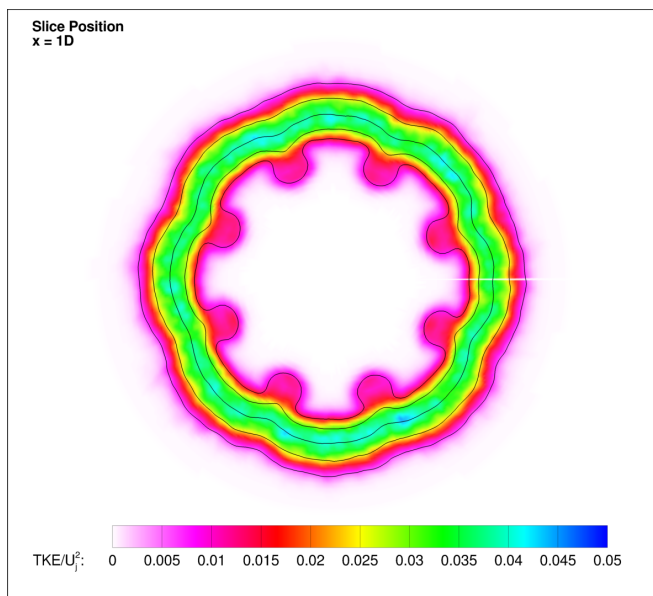

(f) Microjet Case $K / U_{j}^{2}$ at $x=1.0 D_{j}$

Figure 9. Contours of turbulent kinetic energy with lines of axial velocity for clean jet and microjet case 


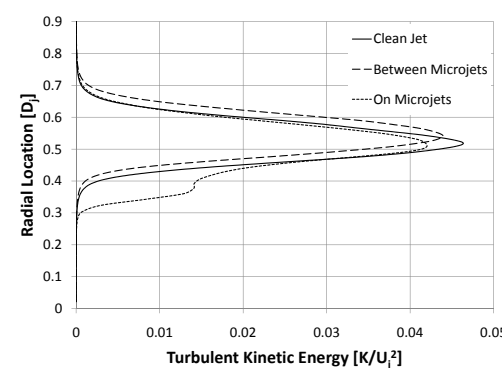

(a) $x / D_{j}=1.0$

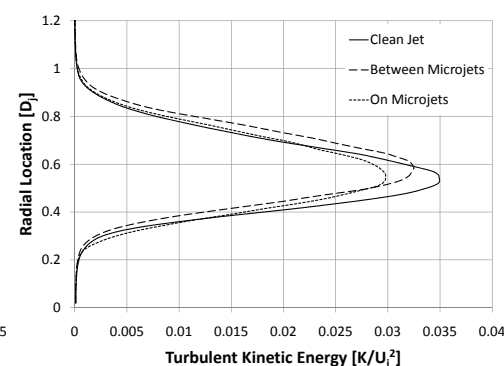

(b) $x / D_{j}=2.5$

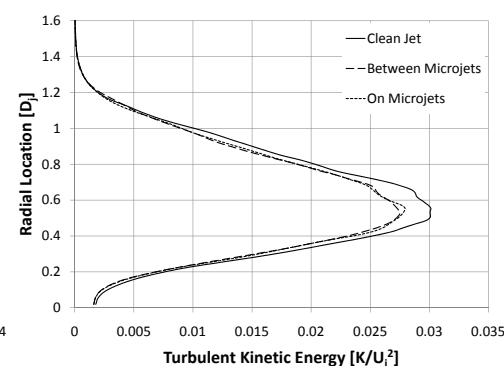

(c) $x / D_{j}=5.0$

Figure 10. Radial profiles of relative turbulent kinetic energy for clean and microjet cases

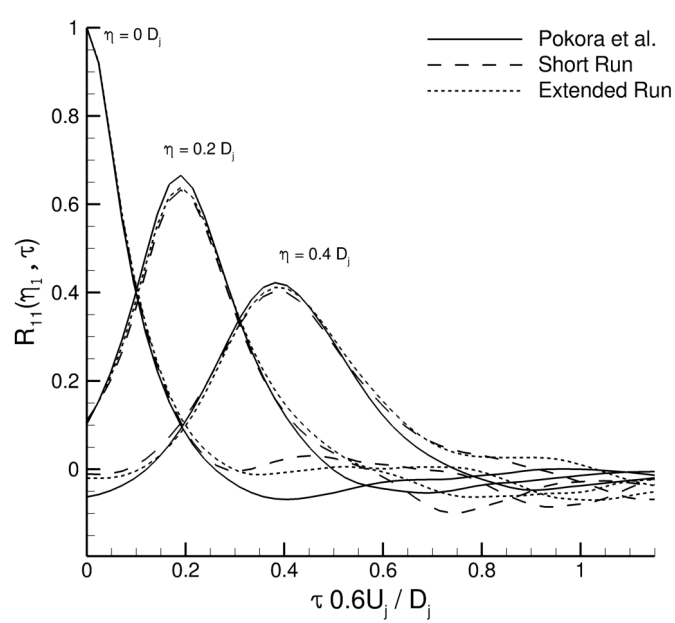

(a) R11 at $x=1.5 D_{j}$

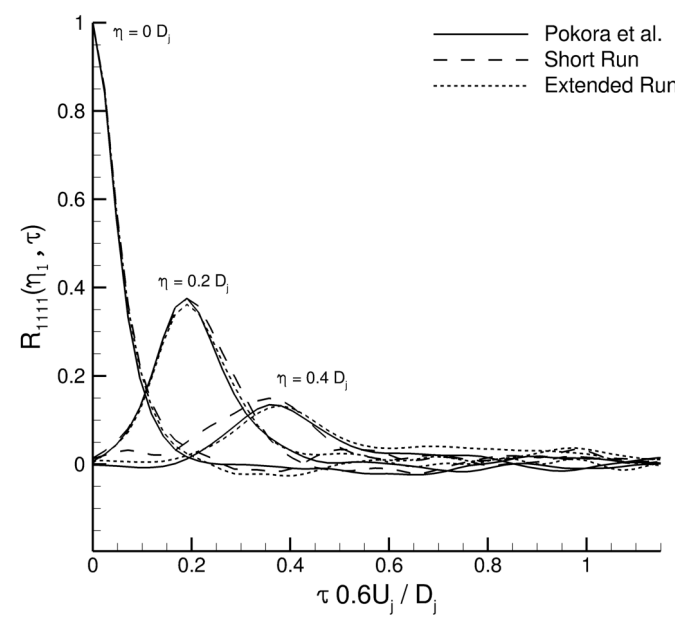

(b) R1111 at $x=1.5 D_{j}$

Figure 11. Clean jet simulation correlation functions of axial velocity at $x / D_{j}=1.5$

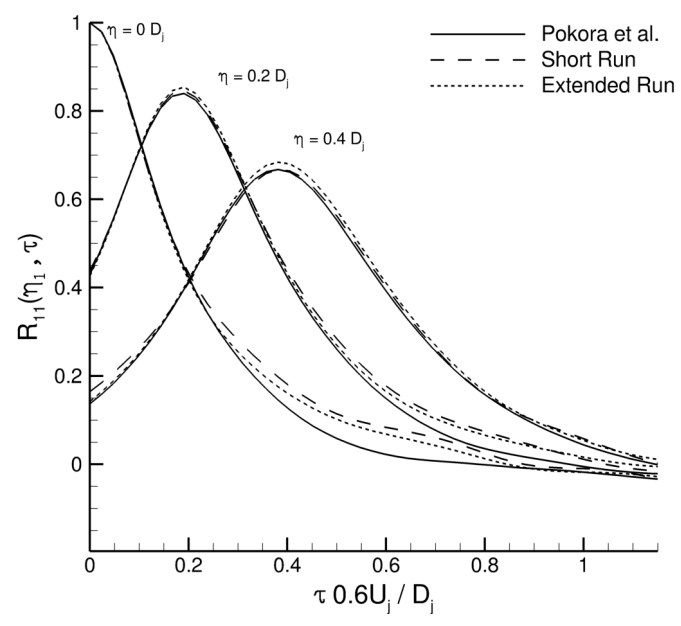

(a) R11 at $x=4.0 D_{j}$

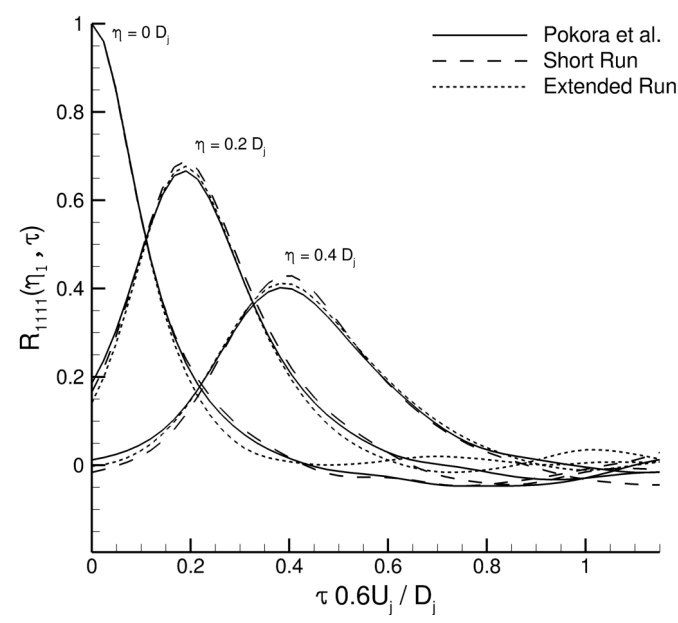

(b) R1111 at $x=4.0 D_{j}$

Figure 12. Clean jet simulation correlation functions of axial velocity at $x / D_{j}=4.0$ 
values of $R_{22}$ and $R_{33}$ are significantly smaller than that of $R_{11}$. This is a indication of the anisotropy of the Reynolds stresses of the jet and has been noted before by Pokora. ${ }^{17}$ Table 2 shows the amplitudes of the six main fourth order correlations, normalised by $R_{1111}$, from the results of Pokora, ${ }^{17}$ the extended run simulation, and Pokora et al. No fourth order correlation amplitude data is available from HarperBourne's experiment. Again, the simulations show an over-prediction of the correlation values compared to the Pokora's experimental data. The simulations can be expected to overpredict the correlation amplitudes because the sparial filtering inherent to the simulation will remove the smaller scale turbulence, leaving the coherent, large scale, energy carrying structures to dominate the flow. This will cause an inflation of the correlation amplitudes over experimental results. Despite the differences in exact values, it is clear from Tables 1 and 2 that the overall trend of the correlation amplitudes is captured by the simulations. The table shows a strong agreement between the numerical results. From the above it appears that the extended run is sufficiently long for high order correlation convergence.

\begin{tabular}{|c|c|c|c|c|c|c|}
\hline & $R_{11}$ & $R_{12}$ & $R_{13}$ & $R_{22}$ & $R_{23}$ & $R_{33}$ \\
\hline Harper-Bourne $^{\mathbf{7}}$ & 1 & - & - & 0.61 & - & 0.80 \\
\hline Pokora $^{\mathbf{1 7}}$ & 1 & 0.30 & $-40 \times 10^{-3}$ & 0.42 & $30 \times 10^{-3}$ & 0.67 \\
\hline Pokora et al. $^{\mathbf{1 6}}$ & 1 & 0.45 & $135 \times 10^{-6}$ & 0.63 & $12.3 \times 10^{-3}$ & 0.81 \\
\hline Extended Run & 1 & 0.44 & $-38 \times 10^{-3}$ & 0.60 & $7.6 \times 10^{-3}$ & 0.79 \\
\hline
\end{tabular}

Table 1. Comparison of relative $2^{\text {nd }}$ order correlation amplitudes

\begin{tabular}{|c|c|c|c|c|c|c|}
\hline & $R_{1111}$ & $R_{1112}$ & $R_{1212}$ & $R_{1313}$ & $R_{2222}$ & $R_{3333}$ \\
\hline Pokora $^{\mathbf{1 7}}$ & 1 & 0.26 & 0.23 & 0.4 & 0.18 & 0.56 \\
\hline${\text { Pokora } \text { et al. }^{\mathbf{1 6}}}^{\text {Extended Run }}$ & 1 & 0.403 & 0.397 & 0.453 & 0.420 & 0.714 \\
\hline Exten & 0.405 & 0.382 & 0.413 & 0.369 & 0.692 \\
\hline
\end{tabular}

Table 2. Comparison of relative $4^{t h}$ order correlation amplitudes

Figures 13, 14, and 15 show the second and fourth order correlation distributions $\left(R_{11}\right.$ and $\left.R_{1111}\right)$ for the clean and microjet cases at $x / D_{j}=1.5,4.0$, and 6.5 , respectively. In all cases, these correlations are the result of an ensemble of eight statistically independent slices containing 2,000 subsamples each, resulting in 16,000 samples for the calculations. At $1.5 D_{j}$ downstream the $\eta=0.2 D_{j}$ and $0.4 D_{j}$ peaks decay less than the baseline clean jet both between and on the microjets in the second and fourth order correlations. This indicates that the correlations in the microjet case are more highly correlated. For the second order correlation the peak value of the correlation between the microjets occurs slightly earlier than the clean jet at $0.2 D_{j}$ separation, showing an increase in convection velocity. This is increased for a separation of $0.4 D_{j}$. Similarly, the convection velocity is seen to be reduced in line with the microjets for both second and fourth order correlations. Between and on the microjets the correlations straddle the clean jet curves after the peak, with the correlations between the microjets falling off before the clean jet. This indicates that for a given separation vector, $\vec{\eta}$, the flow structure is slightly more organised behind the microjets than the clean jet or between the microjets. This is to be expected as the vortex pairs formed behind the microjets will have a more coherent structure than the random turbulence found in the other cases and thus influence the correlations.

At $4.0 D_{j}$ downstream, the correlations show similar convection speeds to those of the clean jet in both second and fourth order correlations. The only significant difference at this station is a reduction in amplitude between the microjets compared to the other cases. Interestingly, at $x / D_{j}=6.5$ the amplitude of the second order correlation distributions for the microjet case are larger than the clean jet, both between and in line with the microjets. The fourth order correlations at this final axial station show less difference from the clean jet, though between the microjets the peak values of the correlations still remains larger.

The relative amplitude of the second and fourth order correlations at three axial location are shown in Figures 16 and 17, respectively. The values of the correlation amplitudes from the microjet cases were normalised by the corresponding $R_{11}$ or $R_{1111}$ of the clean jet at that same axial location. From Figure 16 it is clear that the inclusion of microjets has reduced the relative amplitudes of the correlations, most notably $R_{22}$ and $R_{33}$, despite increasing the values of $R_{11}$ at $x / D_{j}=6.5$. Interestingly, the correlation amplitudes 


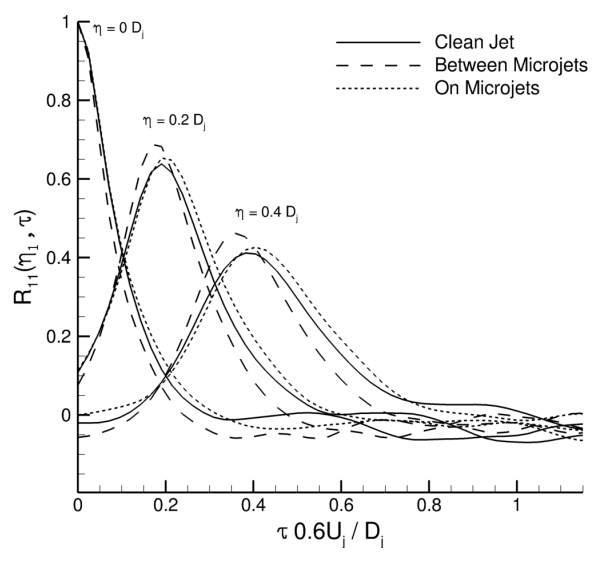

(a) R11 at $x=1.5 D_{j}$

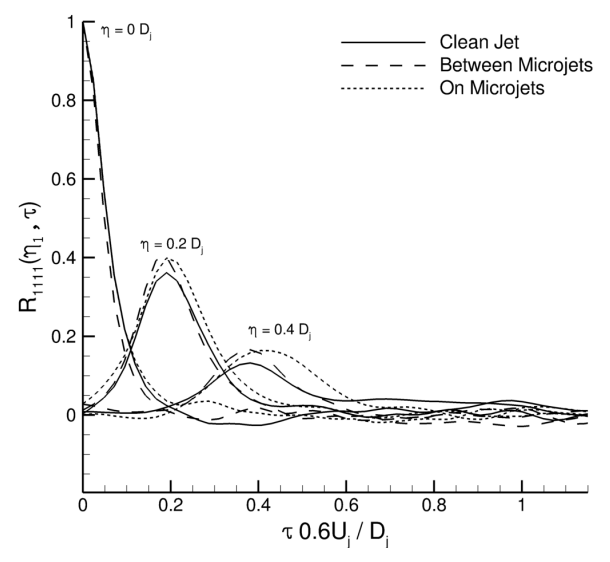

(b) R1111 at $x=1.5 D_{j}$

Figure 13. Correlation functions of axial velocity at $x / D_{j}=1.5$ for clean and microjet cases

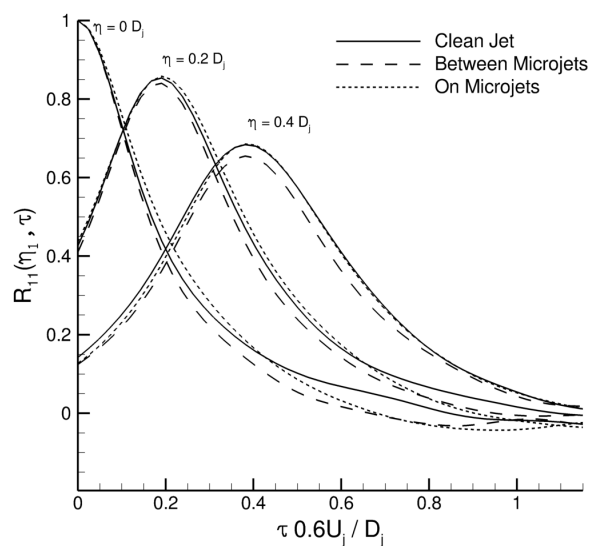

(a) R11 at $x=4.0 D_{j}$

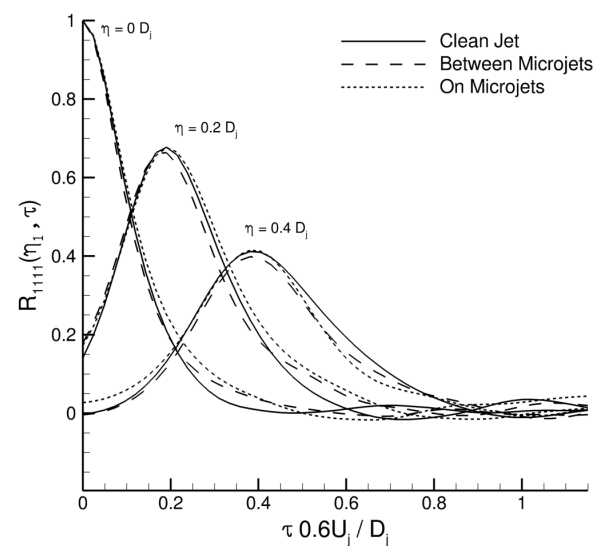

(b) R1111 at $x=4.0 D_{j}$

Figure 14. Correlation functions of axial velocity at $x / D_{j}=4.0$ for clean and microjet cases

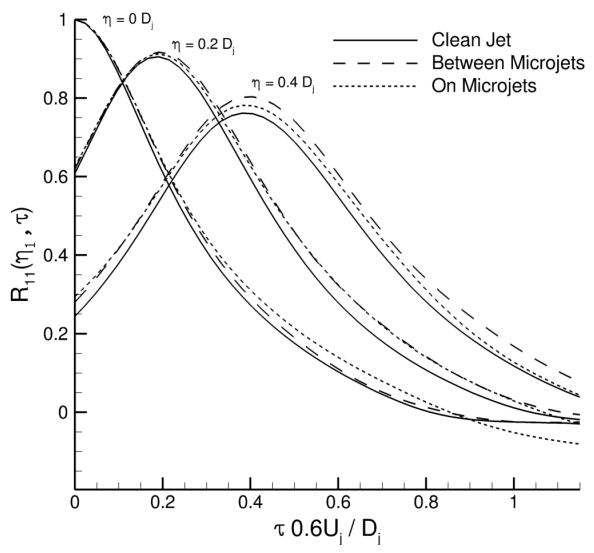

(a) R11 at $x=6.5 D_{j}$

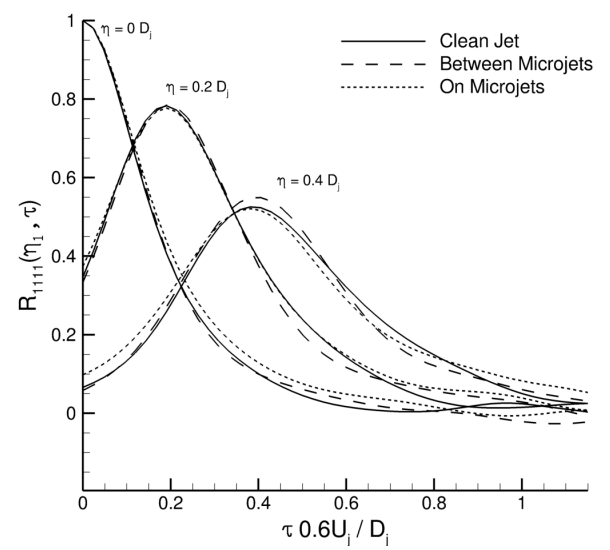

(b) R1111 at $x=6.5 D_{j}$

Figure 15. Correlation functions of axial velocity at $x / D_{j}=6.5$ for clean and microjet cases 


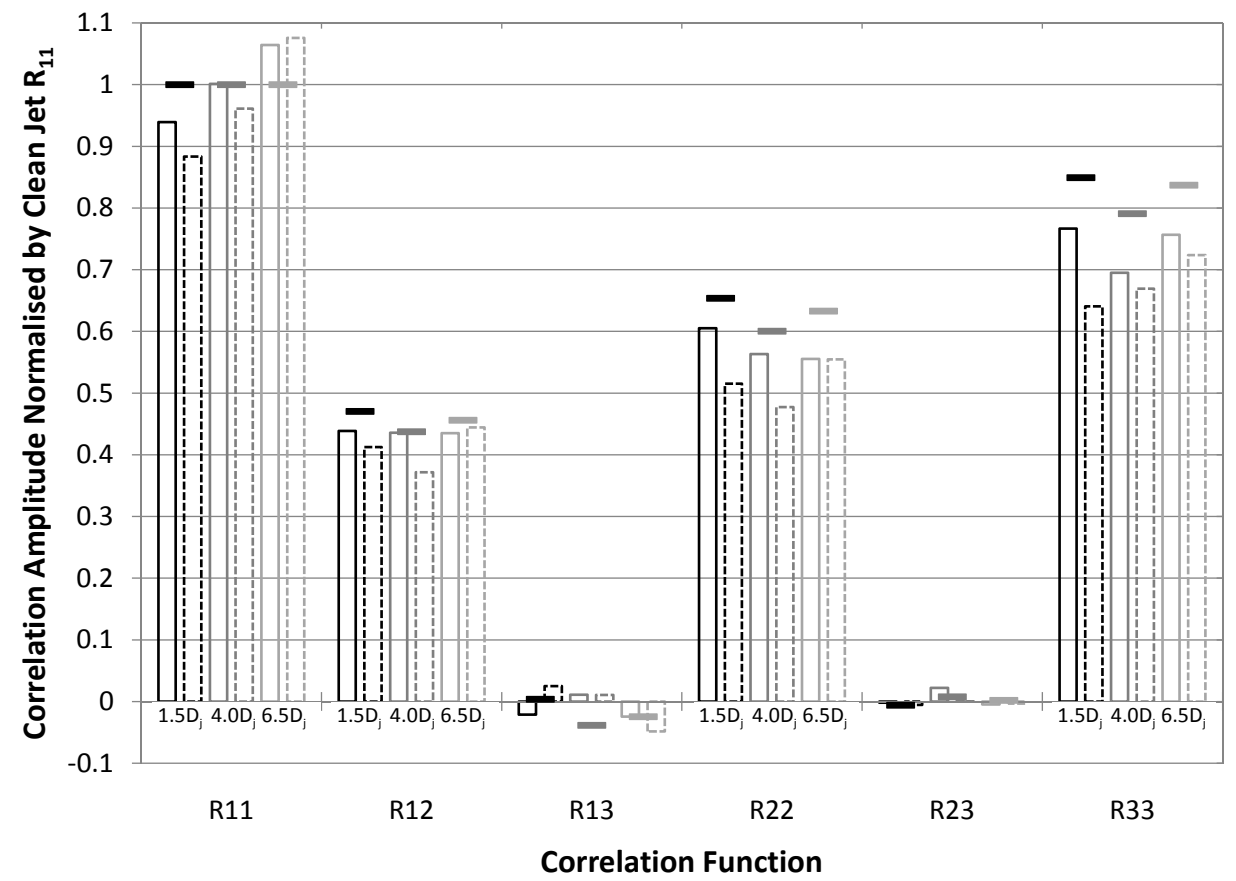

Figure 16. Relative amplitudes of second order correlations. Solid outline vertical bars: in line with the microjets; dashed outline vertical bars: between the microjets; solid horizontal bars: clean jet.

experience the greatest reduction between the microjets, not in line with the microjets as may be expected. The difference in amplitude between the two microjet cases is most noticeable at $1.5 D_{j}$ for $R_{22}$ and $R_{33}$.

Looking at Figure 17 the effect of the microjets on the fourth order correlations can be examined. There is a clear reduction of $R_{2222}$ and $R_{3333}$ due to the introduction of microjets. Other correlation functions depict a more subtle reduction in amplitude. Interestingly, the value of $R_{1111}$ jumps considerably at $x / D_{j}=6.5$ for the microjet case, yet a reduction in other correlation amplitudes is still seen at this axial location. It should be noted that the accuracy at $x / D_{j}=6.5$ will not be as high at earlier stations as the timescales are larger further downstream.

Finally, the streamwise and transverse Eulerian integral lengthscales calculated from the correlations at $x / D_{j}=4.0$ are presented in Table 3 for the clean and microjets cases. These lengthscales are calculated using Equation 4, though the integration is carried out to the first zero crossing. A slight underprediction of the lengthscales is seen, compared to Pokora's ${ }^{17}$ experimental work. The table shows that the lengthscales are reduced compared to the clean jet case both between and in line with the microjets. Again, the larger reduction is seen between the microjets. This indicates a reduction in turbulent structure size around the entire microjet fitted case.

The reduction of major fourth order correlation amplitudes, coupled with smaller turbulent lengthscales, shows promising evidence as to how microjets provide noise reduction when fitted to clean jets. Interestingly, the effects of the microjets appears to diminish by the end of the potential core when looking at single point statistics. However, investigation of two-point, two-time correlations reveals that the effect of the microjets is still present at later axial locations.

\begin{tabular}{|c||c|c|}
\hline & ${ }^{1} L_{11} / D_{j}$ & ${ }^{2} L_{11} / D_{j}$ \\
\hline Pokora $^{\mathbf{1 7}}$ & 0.22 & 0.113 \\
\hline Clean Jet & 0.192 & 0.0864 \\
\hline Between Microjets & 0.1824 & 0.082 \\
\hline On Microjets & 0.1894 & 0.0854 \\
\hline
\end{tabular}

Table 3. Comparison of Eulerian integral lengthscales at $x / D_{j}=4.0$ 


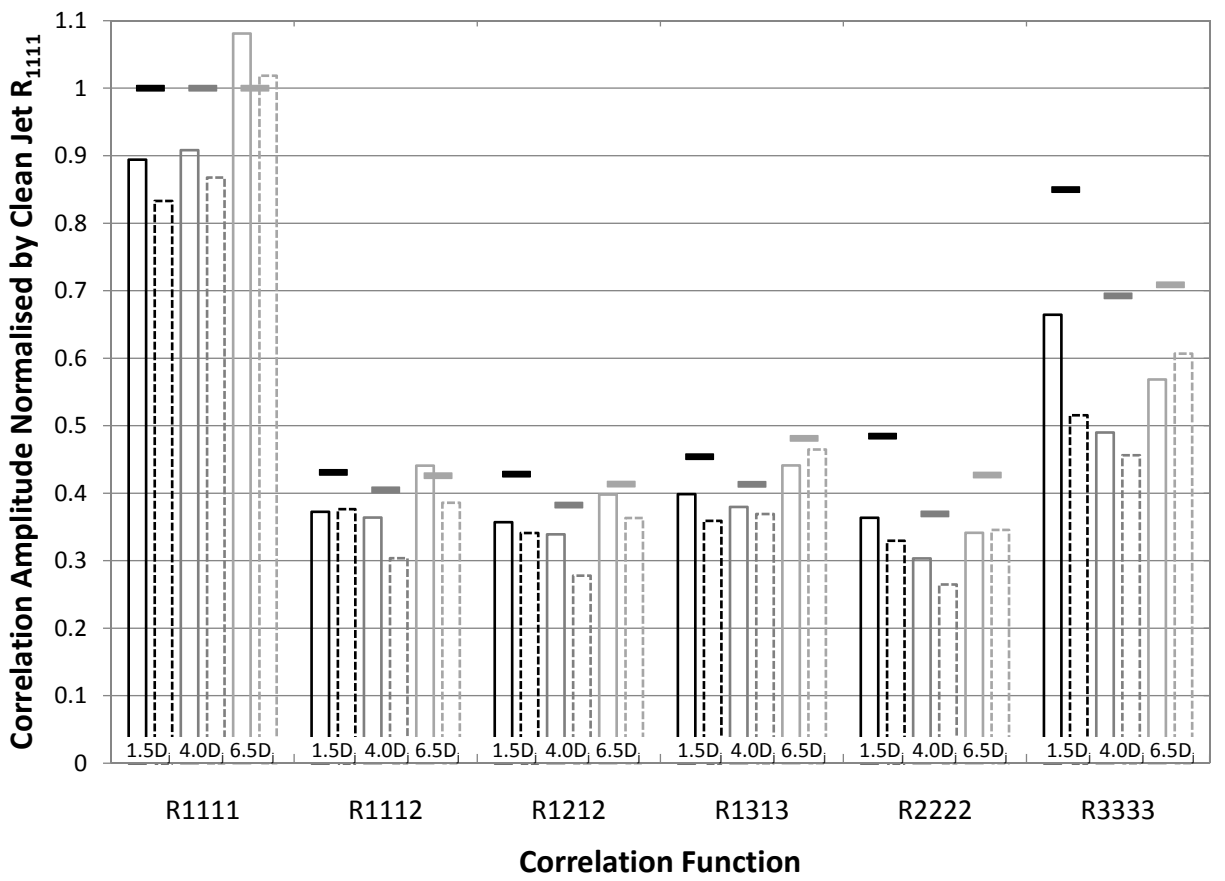

Figure 17. Relative amplitudes of fourth order correlations. Solid outline vertical bars: in line with the microjets; dashed outline vertical bars: between the microjets; solid horizontal bars: clean jet.

\section{Conclusions}

Two large eddy simulations of a high Reynolds number Mach 0.75 isothermal jet, one case including eight microjets, have been performed. The introduction of microjets to the jet created localised regions of low velocity and increased turbulent kinetic energy behind the microjet injection points and on the high speed side of the shear layer. Furthermore, the microjets cause the jet plume to thicken between the microjets, shifting the location of the centre of the shear layer radially outwards. At $x / D_{j}=0.25$ the effect of the microjets is to transform the circular shear layer into a clearly defined octagonal shape, with the microjet inlet points at the corners. This is expected to be a result of the shed vortex pairs inducing a velocity on the otherwise circular shear layer. By the end of the potential core, the microjets have no visible effect on the single point flow field variables. Two-point two-time correlations were extracted from the clean jet and compared to earlier works, showing strong agreement. The spatio-temporal correlation results of the microjet case show distinct differences compared to those of the clean jet. The correlations from the microjet case are seen to be sustained for a longer period of time. The microjets have been shown to reduce the correlation amplitudes both between and in line with the microjets. As the fourth order correlations play an important role in sound generation, a reduction in amplitude indicates the microjets' ability to reduce jet noise. Furthermore, the microjets reduce the turbulent lengthscales, indicating a shift to smaller turbulent eddies. Interestingly, although the effects of the microjets appear to diminish by the end of the potential core when looking at mean flow variables, their presence still has an effect on the noise generating properties of the jet at late axial locations.

The constraints placed on the microjet simulation as a result of using a grid intended for clean round jet simulation must be taken into account. The low number of cells comprising the microjet inlets, coupled with the spatial resolution downstream of the microjets, limits the fidelity of the simulation. Nevertheless, encouraging results are found. Future simulations on finer, bespoke meshes are expected to build on these results. 


\section{Acknowledgments}

Computing time for these calculations was provided by the UK Turbulence Consortium under funding from the EPSRC grant EP/G069581/1.

\section{References}

${ }^{1}$ Åberg, M., Szasz, R.Z., Fuchs, L., and Gutmark, E., "Numerical Study of Fluidic Injection for Noise Reduction," AIAA 2007-11, 45th AIAA Aerospace Sciences Meeting and Exhibit, Reno, Nevada, 8-11 January 2007.

${ }^{2}$ Alkislar, M.B., "Aeroacoustics of a Mach 0.9 Jet with Chevron-Microjet Combination," AIAA 2008-3041, 14th AIAA/CEAS Aeroacoustics Conference, Vancouver, Canada, 5-7 May 2008.

${ }^{3}$ Alkislar, M.B., Krothapalli, A., and Butler, G.W., The Effect of Streamwise Vortices on the Aeroacoustics of a Mach 0.9 Jet, Journal of Fluid Mechanics, Vol 578, pp 139-169, 2007.

${ }^{4}$ Bridges, J. and Brown, C.A., "Parametric Testing of Chevrons on Single Flow Hot Jets", AIAA 2004-2824, 10th Aeroacoustics Conference, Manchester, UK, 10-12 May 2004.

${ }^{5}$ Castelain, T., Sunyach, M., Juvé, D., and Béra, J.-C., Jet-Noise Reduction by Impinging Microjets: An Acoustic Investigation Testing Microjet Parameters, AIAA Journal, Vol 46, pp 1081-1087, 2008.

${ }^{6}$ Jordan P, Gervais Y, Valiŕe J-C, Foulon H. Final results from single point measurements. Project deliverable D3.4, JEAN EU 5th Framework Programme, G4RD-CT2000-00313, 2002.

${ }^{7}$ Harper-Bourne, M., "Jet Noise Turbulence Measurements," AIAA Paper 2003-3214, 9th AIAA/CEAS Aeroacoustics Conference, Hilton Head, SC, 12-14 May 2003.

${ }^{8}$ Huet, M., Fayard, B., Rahier, G., and Vuillot, F., "Numerical Investigation of the Micro-Jets Efficiency for Jet Noise Reduction," AIAA 2009-3127, 15th AIAA/CEAS Aeroacoustics Conference, Miami, Florida, 11-13 May 2009.

${ }^{9}$ Karabasov, S.A., Afsar, M.Z., Hynes, T.P., Dowling, A.P., McMullan, W.A., Pokora, C.D., Page, G.J., and McGuirk, J.J., "Using Large Eddy Simulation within an Acoustic Analogy Approach for Jet Noise Modelling," AIAA 2008-2985, 14th AIAA/CEAS Aeroacoustics Conference, Vancouver, Canada, 5-7 May 2008.

${ }^{10}$ Karabasov, S.A., Afsar, M.Z., Hynes, T.P., Dowling, A.P., McMullan, W.A., Pokora, C.D., Page, G.J., and McGuirk, J.J., Jet Noise: Acoustic Analogy Informed by Large Eddy Simulation, AIAA Journal, Vol 48, pp 1312-1325, 2010.

${ }^{11}$ Lew, P.-T., Najafiyazdi, A., and Mongeau, L., "Unsteady Numerical Simulation of a Round Jet with Impinging Microjets for Noise Suppression," AIAA 2010-18, 48th AIAA Aerospace Sciences Meeting Including the New Horizons Forum and Aerospace Exposition, Orlando, Florida, 4-7 January 2010.

${ }^{12}$ Lighthill, M.J., "On Sound Generated Numerically I. General Theory," Proc. Roy. Soc. Lond., 1952.

${ }^{13}$ Lighthill, M.J., "On Sound Generated Numerically II. Turbulence as a Source of Sound," Proc. Roy. Soc. Lond., 1954.

${ }^{14}$ Liu, J., Kailasanath, K., Ramamurti, R., Munday, D., and Gutmark, E., "Large-Eddy Simulations of a Supersonic Jet with Fluidic Injection for Noise Reduction," AIAA 2010-4024, 16th AIAA/CEAS Aeroacoustics Conference, Stockholm, Sweden, 7-9 June 2010.

${ }^{15}$ McMullan, W.A., Pokora, C.D., Page, G.J., McGuirk, J.J., "Large Eddy Simulation of a High Reynolds Number Subsonic Turbulent Jet for Acoustic Source Capture," AIAA 2008-2974, 14th AIAA/CEAS Aeroacoustics Conference, Vancouver, Canada, 5-7 May 2008.

${ }^{16}$ Pokora, C.D., McMullan, W., Page, G.J., McGuirk, J., "Influence of a Numerical Boundary Layer Trips Within LES of a Subsonic Jet on Spatio-Temporal Correlations," 17th AIAA/CEAS Aeroacoustics Conference, Portland, Oregon, 5-8 June 2011. (Submitted for publication)

${ }^{17}$ Pokora, C.D., "Spatio-Temporal Correlations of Jets using High-Speed Particle Image Velocimetry", Ph.D. thesis, Loughborough University, 2009.

${ }^{18}$ Pokora, C.D., McGuirk, J.J., "Spatio-Temporal Turbulence Correlations using High-Speed PIV in an Axisymmetric Jet", AIAA 2008-3028, 14th AIAA/CEAS Aeroacoustics Conference, Vancouver, Canada, 5-7 May 2008. 